\title{
IDO decreases glycolysis and glutaminolysis by activating GCN2K, while it increases fatty acid oxidation by activating AhR, thus preserving CD4+ T-cell survival and proliferation
}

\author{
THEODOROS ELEFTHERIADIS, GEORGIOS PISSAS, VASSILIOS LIAKOPOULOS and IOANNIS STEFANIDIS
}

Department of Nephrology, Faculty of Medicine, University of Thessaly, 41110 Larissa, Greece

Received December 4, 2017; Accepted March 15, 2018

DOI: $10.3892 / \mathrm{ijmm} .2018 .3624$

\begin{abstract}
It is generally hypothesized in the literature that indoleamine 2,3-dioxygenase (IDO), by degrading L-tryptophan along the kynurenine pathway, suppresses CD4+ T-cell function by inducing apoptosis, inhibiting proliferation and promoting differentiation towards a regulatory phenotype. These effects are either accompanied or directly lead to alterations in cell metabolism. The present study evaluated the pathways that govern the effect of IDO on the utilization of the three main energy sources in CD4+ T-cells. Two-way mixed lymphocyte reactions were performed with or without oleate and/or the IDO inhibitor 1-methyl-DL-tryptophan. In addition, isolated CD4+ T-cells cultured in an oleate-containing medium were activated in the presence or not of the general control nonderepressible 2 kinase (GCN2K) activator tryptophanol. L-tryptophan, glucose and free fatty acid consumption, cell proliferation, apoptosis and the levels of key proteins involved in IDO-mediated signal transduction, and glucose, glutamine and free fatty acid utilization were assessed. The results indicate that IDO decreased glycolysis and glutaminolysis by activating GCN2K, resulting in activation of AMP-activated protein kinase (AMPK). In parallel with AMPK activation, IDO-induced activation of aryl hydrocarbon receptor increased the expression of all carnitine palmitoyltransferase I isoenzymes, leading ultimately to increased free fatty acid oxidation and preservation of CD4+ T-cell survival and proliferation. Thus, contrary to what is generally hypothesized, in a normal environment containing fatty acids, the immunosuppressive effect of IDO may not be due to a decrease in CD4+ T-cell survival and proliferation, since IDO supplies the required
\end{abstract}

Correspondence to: Professor Theodoros Eleftheriadis, Department of Nephrology, Faculty of Medicine, University of Thessaly, Neo Ktirio, Mezourlo Hill, 41110 Larissa, Greece E-mail: teleftheriadis@yahoo.com

Key words: indoleamine 2,3-dioxygenase, aryl hydrocarbon receptor, free fatty acids, T-cells, proliferation, apoptosis energy for cell survival and proliferation by increasing free fatty acid oxidation.

\section{Introduction}

During immune response, indoleamine 2,3-dioxygenase (IDO) is upregulated in antigen presenting cells (APCs) and degrades L-tryptophan along the kynurenine pathway. Depletion of L-tryptophan in the local microenvironment activates the amino-acid sensor general control nonderepressible 2 kinase (GCN2K) in CD4+ T-cells (1-4). According to some, albeit not all, investigators (1-5), L-tryptophan depletion also inhibits the other amino-acid sensor, the mammalian target of rapamycin complex 1 (mTORC1). In parallel, the produced kynurenine activates the aryl hydrocarbon receptor (AhR) $(6,7)$. It is generally thought that through these pathways IDO suppresses CD4+ T-cell function by inducing apoptosis, inhibiting proliferation and promoting differentiation towards a regulatory T-cell lineage (8).

Cell metabolism governs the fate of activated CD4+ T-cells. These cells rely on aerobic glycolysis and glutaminolysis for energy and building blocks required for their survival, proliferation and differentiation towards an effector cell lineage (9-11). However, under conditions that favor differentiation towards regulatory T-cells (Tregs), naïve CD4+ T-cells rely on free fatty acid oxidation in order to cover their energy demands. Notably, blockage of fatty acid oxidation inhibits naïve CD4+ T-cells differentiation towards a regulatory phenotype $(11,12)$.

IDO affects the metabolism of CD4+ T-cells. By upregulating tumor suppressor protein p53 (p53) and downregulating MYC proto-oncogene (c-Myc), IDO alters the expression of key enzymes resulting in suppressed utilization of glucose and glutamine $(3,13)$. In parallel, at least in part due to energy deprivation, IDO induces CD4+ T-cell apoptosis and inhibits their proliferation $(2,3,13)$. Nevertheless, a culture medium that was free of fatty acids was used in the majority of these studies that evaluated the immunosuppressive properties of IDO. Notably, in a study in which free fatty acids were added in cell cultures, IDO increased free fatty acid oxidation and although it promoted Treg differentiation, it did not induce apoptosis or inhibited proliferation (7). Thus, contrary to what is generally 
hypothesized, it is possible that the immunosuppressive properties of IDO rely predominantly on the promotion of Treg differentiation, and that, under physiological conditions where free fatty acids are always present, IDO does not affect CD4+ T-cell apoptosis and proliferation.

Since IDO is a potent immunosuppressive enzyme able in experimental models to ameliorate autoimmune diseases $(14,15)$, prevent allograft rejection $(16,17)$, protect the semiallogenic fetus from the maternal immune system (18), and facilitate escape of cancer from immunosurveillance (19), it may serve as a useful pharmacological target in the future. Thus, delineating the molecular pathways involved in the immunosuppressive function of IDO is imperative. Accordingly, the aim of the present study was to evaluate further the molecular pathways implicated in IDO-induced alterations of glucose, glutamine and free fatty acid metabolism in CD4+ T-cells, and how they ultimately affect cell apoptosis and proliferation.

For this propose, two-way mixed lymphocyte reactions (MLRs), a well-established model of alloreactivity (20), were performed in the presence or not of the free fatty acid oleate and/or of the IDO inhibitor 1-methyl-DL-tryptophan (1-MT). 1-MT has been successfully used to increase the activity of autoimmune diseases and break the immune tolerance to semiallogenic fetuses and allografts $(15,16,18)$. In MLR-derived CD4+ T-cells, the effect of both IDO-induced pathways, namely the activation of GCN2K and AhR, can be evaluated In addition, another system, free of APCs, was generated. Isolated CD4+ T-cells, cultured in an oleate-containing medium, were activated with anti-CD2/CD3/CD28 covered beads in the presence or not of the GCN2K activator tryptophanol. Tryptophanol is known to activate GCN2K and inhibit CD4+ T-cell proliferation and survival $(1,3,4,13)$. It is expected that in this system tryptophanol would activate only GCN2K, leaving AhR unaffected. Comparing the results from these two experimental systems, the effect of IDO-induced GCN2K activation can be discriminated from the effect of AhR activation on CD4+ T-cells.

\section{Materials and methods}

Subjects. Blood samples were collected from 6 healthy volunteers (4 males and 2 females; age, $35.83 \pm 5.78$ years) in April 2017. A written informed consent was obtained from each individual enrolled in the study. The Ethics Committee of the Faculty of Medicine, University of Thessaly (Larisa, Greece) approved the study protocol. The number of approval is $558 / 10-2-2017$.

Peripheral blood mononuclear cells (PBMCs) and CD4+ $T$-cell isolation and culture. PBMCs were isolated from whole blood by Ficoll-Hypaque density gradient centrifugation (Histopaque 1077; Sigma-Aldrich; Merck KGaA, Darmstadt, Germany) and counted by optical microscopy on a Neubauer plaque. Cell viability was assessed by trypan blue assay (Sigma-Aldrich; Merck KGaA).

PBMCs were resuspended in RPMI-1640 medium with L-glutamine and $10 \mathrm{mM}$ 4-(2-hydroxyethyl)-1-piperazineethanesulfonic acid (HEPES) (Sigma-Aldrich; Merck KGaA) and supplemented with $10 \%$ fetal bovine serum (Sigma-Aldrich; Merck KGaA) and antibiotic-antimycotic solution (Sigma-Aldrich; Merck KGaA). Then, PBMCs from different individuals were seeded in couples in order to set up ten different two-way MLRs. Unless otherwise stated, oleate (Sigma-Aldrich; Merck KGaA) was added in all experiments to the culture medium at a concentration of $0.8 \mathrm{mM}$. Oleate was selected because this free fatty acid is not cytotoxic for human cells at a concentration up to $1 \mathrm{mM}(21)$.

In the case of experiments with the GCN2K activator tryptophanol (Sigma-Aldrich; Merck KGaA), CD4+ T-cells were isolated from fresh PMBCs using the Human CD4+ T-Cell Isolation kit (Miltenyi Biotec $\mathrm{GmbH}$, Bergisch Gladbach, Germany). Isolated CD4+ T-cells were cultured in the same medium as the PBMCs supplemented with $0.8 \mathrm{mM}$ oleate. The experiment was repeated six independent times. All cultures were incubated at $37^{\circ} \mathrm{C}$ in a humidified atmosphere containing $5 \% \mathrm{CO}_{2}$.

L-tryptophan, glucose and oleate consumption. MLRs were performed in 12-well plates for 7 days in the presence or not of the IDO inhibitor 1-MT (100 $\mu \mathrm{M}$; Sigma-Aldrich; Merck KGaA). The concentration of 1-MT was selected according to previous experiments $(2,3,13)$. The number of PBMCs of each member of the MLR couple was $5 \times 10^{5}$, bringing the total cell number to $1 \times 10^{6}$ cells/MLR. L-tryptophan concentration was assessed in the supernatants using ELISA (cat. no. E01T0140; BlueGene Biotech, Shanghai, China). The sensitivity of the above kit is $1 \mathrm{ng} / \mathrm{ml}$. L-tryptophan consumption was calculated by subtracting the results in the cell culture supernatants from the concentration found in the supplemented culture medium. Similarly, glucose consumption was assessed by using the Element blood glucose monitor (Infopia, Inc., Titusville, FL, USA), and oleate consumption colorimetrically through the Free Fatty Acid Quantification kit (Abcam, Cambridge, UK). The detection limit of the above kit is $2 \mu \mathrm{M}$. Ten such MLRs were performed.

Glucose and oleate consumption were also assessed in activated isolated CD+4 T-cells in the presence or not of tryptophanol. For this propose, isolated CD4+ T-cells from fresh PBMCs were cultured in 12-well plates $\left(1 \times 10^{6}\right.$ cells/well) and activated for $72 \mathrm{~h}$ with anti-CD2/CD3/CD28-conjugated beads using the T-Cell activation/expansion kit (Miltenyi Biotec $\mathrm{GmbH}$ ) in a bead to cell ratio of 1:2 and in the presence or not of $0.25 \mathrm{mM}$ tryptophanol. The above concentration of tryptophanol was selected according to previous studies $(1,3,4,13)$. The experiment was repeated six independent times.

Cell proliferation mixed lymphocyte reactions were performed in 96-well plates for 7 days in the presence or not of $100 \mu M$ 1-MT. The number of PBMCs of each member of the MLR couple was $5 \times 10^{4}$, bringing up the total cell number to $1 \times 10^{5}$ cells per MLR. In parallel, $1 \times 10^{5}$ resting PBMCs from each member were cultured in the same 96-well plate, serving as controls. Cell proliferation was assessed with the Cell Proliferation ELISA (cat. no. 11647229001; Roche Diagnostics, Indianapolis, IN, USA) using bromodeoxyuridine labeling and immunoenzymatic detection, according to the manufacturer's protocol. The proliferation index was calculated as follows: Proliferation index = optical density (OD) derived from each MLR/mean of the ODs derived from the control resting PBMCs cultures of the two subjects that formed the specific 
MLR. Ten such MLRs were performed, each in triplicate and the results refer to the mean of the three measurements.

The proliferation of CD4+ T-cells retrieved from freshly isolated PBMCs was also determined by Cell Proliferation ELISA. Resting, stimulated with T-Cell activation/expansion kit or stimulated in the presence of $0.25 \mathrm{mM}$ tryptophanol CD4+ T-cells were cultured in 96-well plates $\left(1 \times 10^{5}\right.$ cells/well) for $72 \mathrm{~h}$. The proliferation index was calculated as follows: Proliferation index=OD derived from activated CD4+ T-cells/ODs derived from the respective resting CD4+ T-cells. T-cells were derived from six individuals and the experiments were performed in triplicates. The results refer to the mean of the three measurements.

Assessment of proteins of interest in MLR-derived CD4+ T-cells and in isolated CD4+ T-cells activated with anti-CD2/CD3/CD28 covered beads. Ten MLRs were performed in 12-well plates in the presence or not of $100 \mu \mathrm{M}$ 1-MT, with a cellular number of each PBMC population in the MLR context remaining the same as before. At the end of the 7 day period that MLRs lasted, CD4+ T-cells were isolated from the MLRs by negative selection using the Human CD4+ T-Cell Isolation kit. MLR-derived CD4+ T-cells were counted via optical microscopy on a Neubauer plaque and cell viability was determined by trypan blue assay. Equal numbers of T-cells from each MLR were lysed using the T-PER tissue protein extraction reagent (Thermo Fisher Scientific, Inc., Waltham, MA, USA) supplemented with protease (Merck KGaA) and phosphatase (Roche Diagnostics) inhibitors.

Protein was also extracted from freshly isolated and activated CD4+ T-cells. PBMCs were isolated from the blood of six individuals. Following PBMCs isolation, CD4+ T-cells were isolated with the Human CD4+ T-Cell Isolation Kit, cultured in 12-well plates $\left(1 \times 10^{6}\right.$ cells/well) and activated with anti-CD2/CD3/CD28-conjugated beads in the presence or not of $0.25 \mathrm{mM}$ tryptophanol for $72 \mathrm{~h}$. Then they were counted via optical microscopy on a Neubauer plaque, cell viability was determined by trypan blue assay and equal numbers of cells were lysed using the T-PER tissue protein extraction reagent (Thermo Fisher Scientific, Inc.).

Protein was quantified via Bradford assay (Sigma-Aldrich; Merck KGaA) and $10 \mu \mathrm{g}$ from each sample was used for western blotting. For this purpose, sodium dodecyl sulfate (SDS) polyacrylamide 4-12\% Bis-Tris gels (Thermo Fisher Scientific, Inc.) and polyvinylidene fluoride (PVDF) membranes (Thermo Fisher Scientific, Inc.) were used. Blots were incubated with the primary antibody for $16 \mathrm{~h}$ at $4^{\circ} \mathrm{C}$, followed by a $30 \mathrm{~min}$ incubation with the secondary antibody at room temperature (anti-rabbit horseradish peroxidase (HRP)-conjugated IgG; 1:1,000; cat. no. 7074; Cell Signaling Technology, Inc., Danvers, MA, USA). For the primary antibody against cytochrome P450 family 1 subfamily A polypeptide 1 (CYP1A1), a goat anti-mouse HRP-conjugated IgG secondary antibody $(1: 1,000$; cat. no. sc2005; Santa Cruz Biotechnology, Inc., Dallas, TX, USA) was used. In case of reprobing the PVDF blots, the previous primary and secondary antibody were removed with the Restore Western Blot Stripping Buffer (Thermo Fisher Scientific, Inc.) according to the manufacturer's protocol. Analysis of the western blots was performed using the ImageJ software (National Institute of Health, Bethesda, MD, USA).
Primary antibodies targeting the following proteins were used in western blotting: Eukaryotic initiation factor $2 \alpha$ phosphorylated at serine 51 (p-eIF2 $\alpha$; 1:500; cat. no. 9721; Cell Signaling Technology, Inc.) which is the substrate of GCN2K; $70 \mathrm{kDa}$ ribosomal protein S6 kinase phosphorylated at threonine 389 (p-p70S6K; 1:1,000; cat. no. 9234; Cell Signaling Technology, Inc.) which is the substrate of mTORC1; the transcriptional target of AhR, CYP1A1 (1:500; cat. no. sc-25304; Santa Cruz Biotechnology, Inc.); the $\alpha$ subunit of activated AMP-activated protein kinase phosphorylated at threonine 172 (p-AMPK; 1:1,000; cat. no. 2535; Cell Signaling Technology, Inc.); the substrate of AMPK acetyl-CoA carboxylase 2 phosphorylated at serine 221 (p-ACC2; 1:500; cat. no. ab109540; Abcam); the activated cleaved caspase- 3 at aspartate 175 (CC3; 1:500; cat. no. 9664; Cell Signaling Technology, Inc.); glucose transporter-1 (GLUT1; 1:500; cat. no. sc-7903, Santa Cruz Biotechnology, Inc.); hexokinase-II (HKII; 1:1,000; cat. no. 2867; Cell Signaling Technology, Inc.); glutaminase 1 (GLS1; 1:100; cat. no. AP18036PUN; Acris Antibodies GmbH: Origen Europe, Herford, Germany); glutaminase2 (GLS2; 1:100; cat. no. AP17426PU-N; Acris Antibodies GmbH; Origen Europe); carnitine palmitoyltransferase I isoenzyme A (CPT1A; 1:1,000; cat. no. 12252S; Cell Signaling Technology, Inc.); CPT1B (1:1,000; cat. no. ab134988; Abcam); and CPT1C (1:250; cat. no. ab87498; Abcam). All western blot results were normalized to $\beta$-actin (1:5,000; cat. no. 4967; Cell Signaling Technology, Inc.).

Statistical analysis. The SPSS software (version 13; SPSS Inc., Chicago, IL, USA) was used for statistical analysis. The normality of the evaluated variables was assessed and confirmed by the one-sample Kolmogorov-Smirnov test. For comparison of means, paired-sample t-test was used. Results were expressed as mean \pm standard deviation. $\mathrm{P}<0.05$ was considered to indicate a statistically significant difference.

\section{Results}

Effect of free fatty acids on the role of IDO and GCN2K in T-cell proliferation. In the presence of oleate, the IDO inhibitor 1-MT did not affect T-cell clonal expansion in MLRs. The proliferation index was $4.84 \pm 0.29$ in the absence of $1-\mathrm{MT}$ and $4.80 \pm 0.26$ with 1-MT treatment $(\mathrm{P}=0.610$; Fig. 1A). By contrast, in the absence of oleate, 1-MT treatment increased the proliferation index from $2.07 \pm 0.17$ to $3.41 \pm 0.51$ ( $\mathrm{P}<0.001$; Fig. $1 \mathrm{~B})$. Of note, the T-cell proliferation index was significantly higher in MLRs performed in the presence of oleate compared with MLRs performed in the absence of oleate $(\mathrm{P}<0.001$; Fig. 1A and $\mathrm{B})$.

The GCN2K activator tryptophanol decreased proliferation of isolated and activated with anti-CD2/CD3/CD28-conjugated beads CD4+ T-cells despite the presence of oleate. The proliferation index was $5.18 \pm 0.74$ in the absence of tryptophanol and $2.74 \pm 0.36$ with tryptophanol treatment $(\mathrm{P}<0.001$; Fig. $1 \mathrm{C})$. These results demonstrated that IDO suppressed T-cell proliferation only in the absence of free fatty acids, whereas the GCN2K activator tryptophanol inhibited cell proliferation in activated CD4+ T-cells despite the presence of free fatty acids.

Effect of free fatty acids on the role of IDO and GCN2K in T-cell apoptosis. In MLR-derived CD4+ T-cells, and in the 

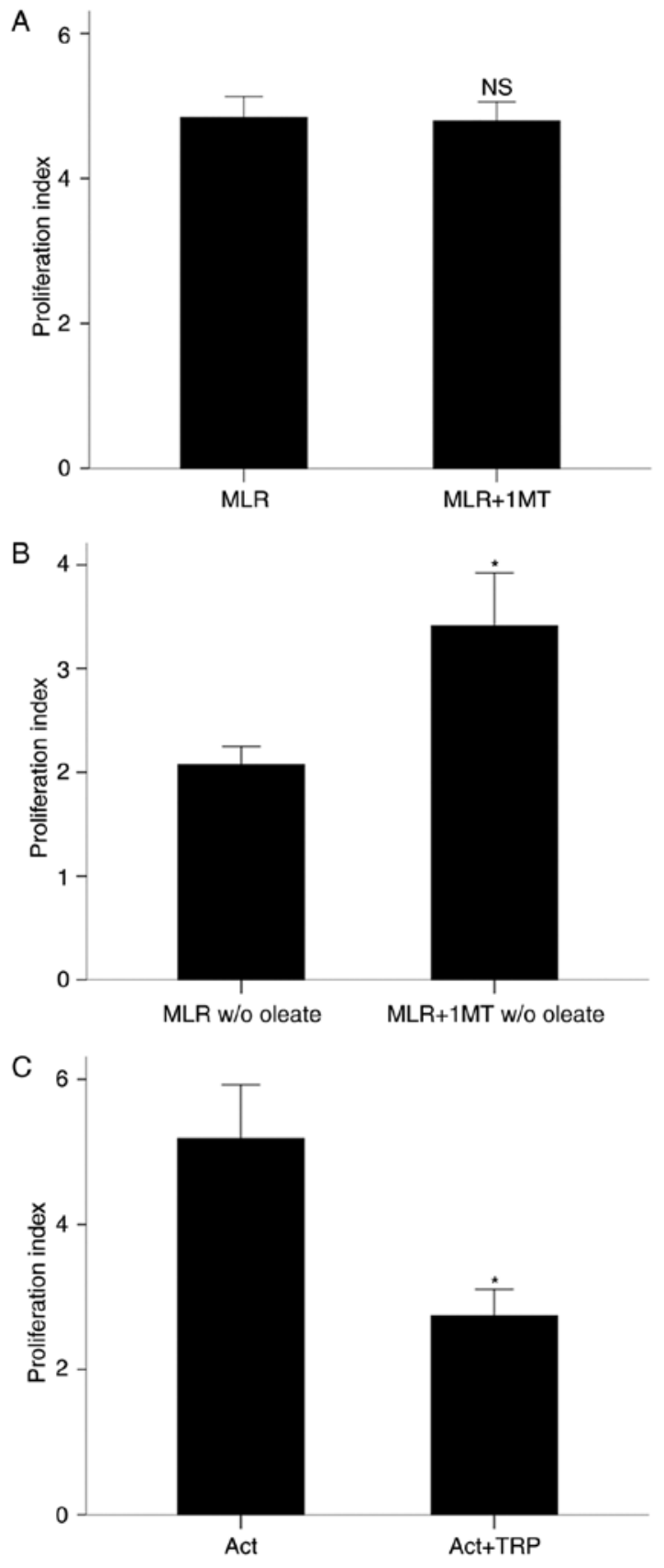

Figure 1. Effect of IDO inhibition and GCN2K activation on cell proliferation in MLRs and activated isolated CD4+ T-cells, respectively. (A and B) PBMCs from different individuals were coupled in 10 different MLRs performed in (A) the presence or (B) absence of oleate and treated with the IDO inhibitor 1-MT. 1-MT did not affect cell proliferation in the presence of oleate but increased it in the absence of oleate. (C) CD4+ T-cells were isolated from the PBMCs of 6 individuals, cultured in an oleate-containing medium and activated with anti-CD2/CD3/CD28-conjugated beads in the presence or absence of the GCN2K activator tryptophanol. Tryptophanol decreased cell proliferation. Results are presented as mean \pm standard deviation. ${ }^{*} \mathrm{P}<0.05$. IDO, indoleamine 2,3-dioxygenase; GCN2K, general control nonderepressible 2 kinase; MLR, mixed lymphocyte reactions; PBMCs, peripheral blood mononuclear cells; TRP, tryptophanol; NS, not significant.

presence of oleate, 1-MT treatment did not affect the protein levels of activated cleaved caspase- 3 compared with the untreated cells $(\mathrm{P}=0.907$; Fig. $2 \mathrm{~A}$ and $\mathrm{B})$. By contrast, when oleate was absent from the culture medium, 1-MT treatment resulted in a decrease of apoptosis in MLR-derived
CD4+ T-cells (Fig. 2A), with the levels of cleaved caspase-3 decreasing to $0.56 \pm 0.05$-fold of the levels in the untreated MLRs (Fig. 2C).

In isolated CD4+ T-cells cultured in an oleate-containing medium and activated with anti-CD2/CD3/CD28-conjugated beads, tryptophanol increased the levels of cleaved caspase- 3 by $1.52 \pm 0.11$-fold compared with the untreated cells $(\mathrm{P}<0.001$; Fig. 2A and D). These results demonstrated that, in MLR-derived CD4+ T-cells, IDO induced apoptosis only in the absence of free fatty acids, whereas in activated isolated CD4+ T-cells the GCN2K activator tryptophanol induced cell apoptosis despite the presence of free fatty acids.

Effect of IDO and GCN2K on L-tryptophan consumption and AhR activation. In MLRs performed in the presence of oleate, the IDO inhibitor 1-MT decreased L-tryptophan consumption from $4.67 \pm 0.43 \mu \mathrm{g} / \mathrm{ml}$ in untreated cells to $1.16 \pm 0.73 \mu \mathrm{g} / \mathrm{ml}$ in 1-MT-treated cells $(\mathrm{P}<0.001$; Fig. 3A). In CD4+ T-cells derived from the above 1-MT-treated MLRs, GCN2K and AhR activity decreased, whereas mTORC1 activity remained unaffected (Fig. 3B). More precisely, 1-MT treatment decreased the levels of the GCN2K substrate p-eIF2 $\alpha$ and of the AhR transcriptional target CYP1A1 to $0.43 \pm 0.13(\mathrm{P}<0.001)$ and $0.46 \pm 0.18(\mathrm{P}<0.001)$ fold relative to the levels in untreated MLRs, respectively (Fig. 3C). By contrast, the levels of the mTORC1 substrate, $\mathrm{p}-\mathrm{p} 70 \mathrm{~S} 6 \mathrm{~K}$, were altered only by a factor of $1.05 \pm 0.14$ ( $\mathrm{P}=0.265$; Fig. 3C).

In isolated CD4+ T-cells cultured in an oleate-containing medium and activated with anti-CD2/CD3/CD28-conjugated beads, tryptophanol, as expected, increased the activity of GCN2K, but not of AhR (Fig. 3B). In specific, p-eIF2 $\alpha$ levels increased by a factor of $2.05 \pm 0.23$ in the tryptophanol-treated cells compared with untreated cells ( $\mathrm{P}<0.001$; Fig. 3D), while CYP1A1 expression was not significantly altered by tryptophanol treatment $(1.01 \pm 0.12$-fold; $\mathrm{P}=0.815$; Fig. 3D). These results demonstrated that IDO increased L-tryptophan consumption and activated GCN2K and AhR, whereas the GCN2K activator tryptophanol did not affect AhR activity in activated isolated CD4+ T-cells.

Effect of IDO and GCN2K on glucose consumption. In MLRs performed in the presence of oleate, the IDO inhibitor 1-MT increased glucose consumption from $105.60 \pm 37.39 \mathrm{mg} / \mathrm{dl}$ in untreated cells to $148.40 \pm 32.01 \mathrm{mg} / \mathrm{dl}$ in 1-MT-treated cells ( $\mathrm{P}<0.001 ;$ Fig. 4A). In isolated CD4+ T-cells cultured in an oleate-containing medium and activated with anti-CD2/CD3/CD28-conjugated beads, the GCN2K activator tryptophanol decreased glucose consumption from $101.00 \pm 12.99 \mathrm{mg} / \mathrm{dl}$ in the untreated cells to $70.67 \pm 12.56 \mathrm{mg} / \mathrm{dl}$ in the tryptophanol-treated cells $(\mathrm{P}<0.001$; Fig. 4B).

Next, the protein expression levels of GLUT1 and HKII were examined, as a measure of glucolysis activity. In CD4+ T-cells derived from 1-MT-treated MLRs, GLUT1 and HKII expression was increased by a factor of $1.91 \pm 0.42(\mathrm{P}<0.001)$ and $1.97 \pm 0.21(\mathrm{P}<0.001)$, respectively, compared with untreated T-cells (Fig. 4C and D). In isolated, cultured in the presence of oleate, and activated with anti-CD2/CD3/CD28-conjugated beads CD4+ T-cells, tryptophanol decreased GLUT1 and HKII expression to $0.43 \pm 0.10(\mathrm{P}<0.001)$ and $0.74 \pm 0.08(\mathrm{P}<0.001)$ fold relative to the levels of the untreated activated CD4+ T-cells, 
A
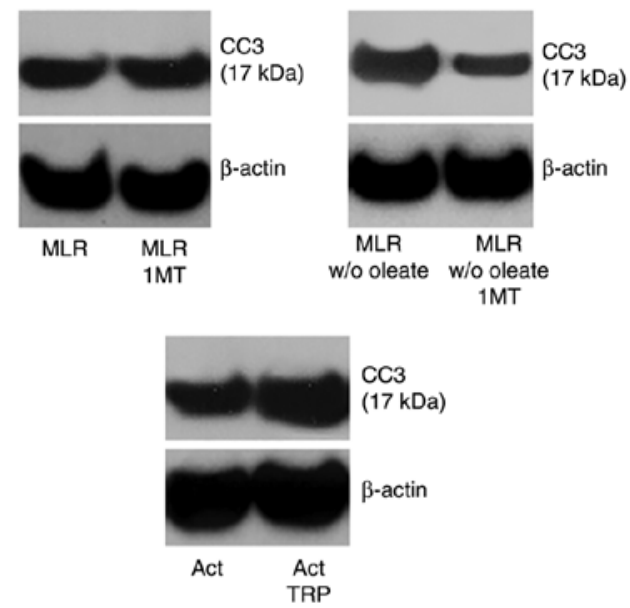

C

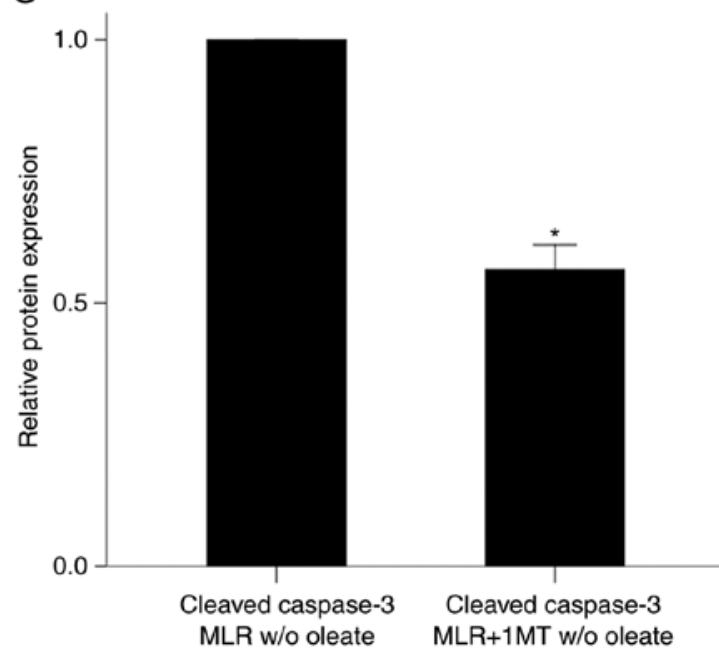

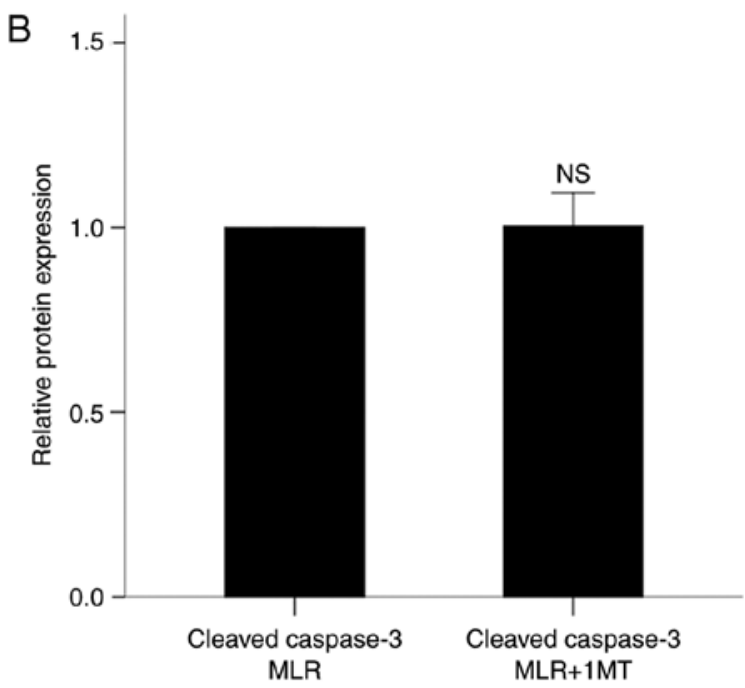

D

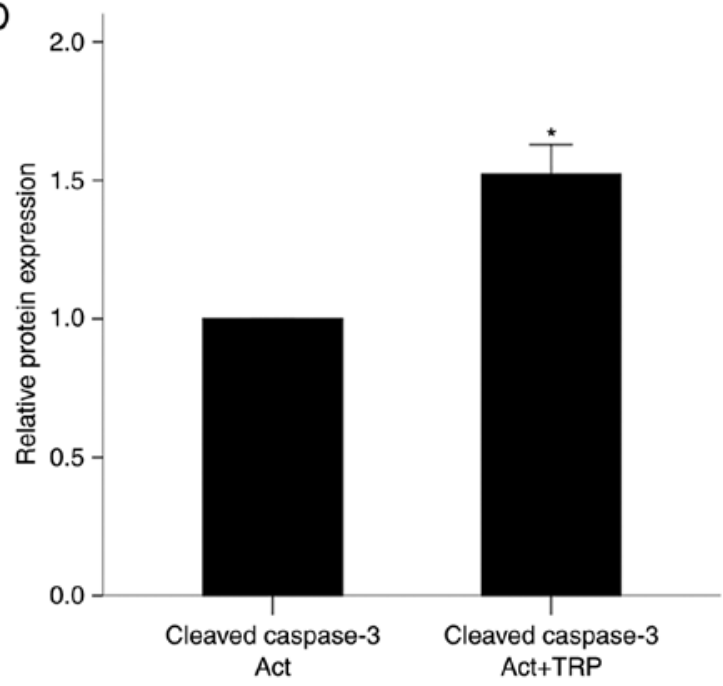

Figure 2. Effect of IDO inhibition and GCN2K activation on cell apoptosis in MLR-derived CD4+ T-cells and activated isolated CD4+ T-cells, respectively. (A) Representative blots from western blot analysis for protein levels of cleaved caspase-3 in experimental groups. (B) PBMCs from different individuals were coupled in 10 different MLRs performed in the presence or (C) absence of oleate and treated with the IDO inhibitor 1-MT. Then, CD4+ T-cells were isolated from the MLRs and protein was extracted for western blotting. 1-MT did not affect the levels of cleaved caspase-3 in MLR-derived CD4+ T-cells cultured with oleate but decreased the levels of cleaved caspase-3 in MLR-derived CD4+ T-cells cultured without oleate. (D) CD4+ T-cells were isolated from the PBMCs of 6 individuals, cultured in an oleate-containing medium and activated with anti-CD2/CD3/CD28-conjugated beads in the presence or absence of the GCN2K activator tryptophanol. Then, the protein was extracted and western blotting revealed that tryptophanol increased cell apoptosis. Results are presented as mean \pm standard deviation. ${ }^{*} \mathrm{P}<0.05$. IDO, indoleamine 2,3-dioxygenase; GCN2K, general control nonderepressible 2 kinase; MLR, mixed lymphocyte reactions; PBMCs, peripheral blood mononuclear cells; CC3, cleaved caspase-3; TRP, tryptophanol; NS, not significant.

respectively (Fig. 4C and E). These results demonstrated that IDO and the GCN2K activator tryptophanol decreased glucose consumption in CD4+ T-cells.

Effect of IDO and GCN2K on the expression of key glutaminolysis-associated enzymes. In CD4+ T-cells derived from MLRs performed in an oleate-containing medium and treated with 1-MT, GLS1 and GLS2 expression increased by a factor of $2.30 \pm 0.26(\mathrm{P}<0.001)$ and $1.40 \pm 0.29(\mathrm{P}<0.001)$, respectively, compared with untreated cells (Fig. 5A and B). In isolated, cultured with oleate, and activated with anti-CD2/CD3/CD28-conjugated beads CD4+ T-cells, tryptophanol treatment decreased GLS1 and GLS2 expression to $0.74 \pm 0.07(\mathrm{P}<0.001)$ and $0.58 \pm 0.11(\mathrm{P}<0.001)$ fold relative to the levels of the untreated activated CD4+ T-cells, respectively (Fig. 5A and C). These results demonstrated that IDO and the GCN2K activator tryptophanol decreased the expression of key enzymes involved in glutaminolysis.

Effect of IDO and GCN2K on free fatty acid consumption and expression of $A M P K$ and CPT1. In MLRs performed in the presence of oleate, the IDO inhibitor 1-MT decreased oleate consumption from $0.49 \pm 0.05 \mathrm{mM}$ in the untreated cells to $0.19 \pm 0.02 \mathrm{mM}$ in the $1-\mathrm{MT}$-treated cells $(\mathrm{P}<0.001$; Fig. $6 \mathrm{~A})$. In isolated $\mathrm{CD} 4+\mathrm{T}$-cells cultured in an oleate-containing medium and activated with anti-CD2/CD3/CD28-conjugated beads, the GCN2K activator tryptophanol did not significantly alter oleate consumption, which remained low both in the absence $(0.15 \pm 0.01 \mathrm{mM})$ and in the presence $(0.16 \pm 0.02)$ of the activator $(\mathrm{P}=0.158$; Fig. $6 \mathrm{~B})$. These findings suggest that although IDO increased free fatty acid consumption, the GCN2K activator tryptophanol had not effect. 
A

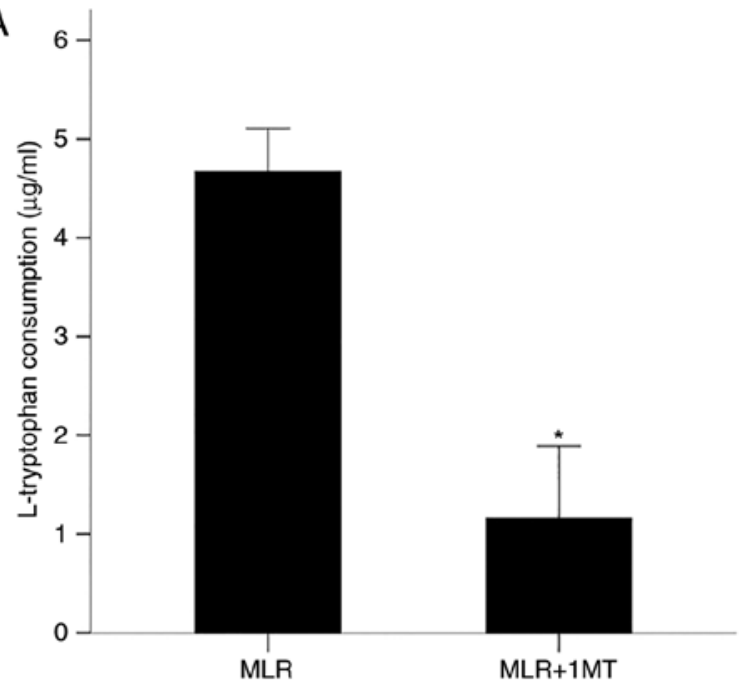

B
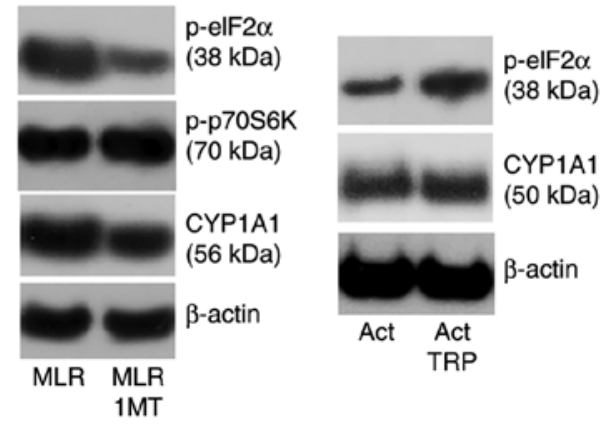

C
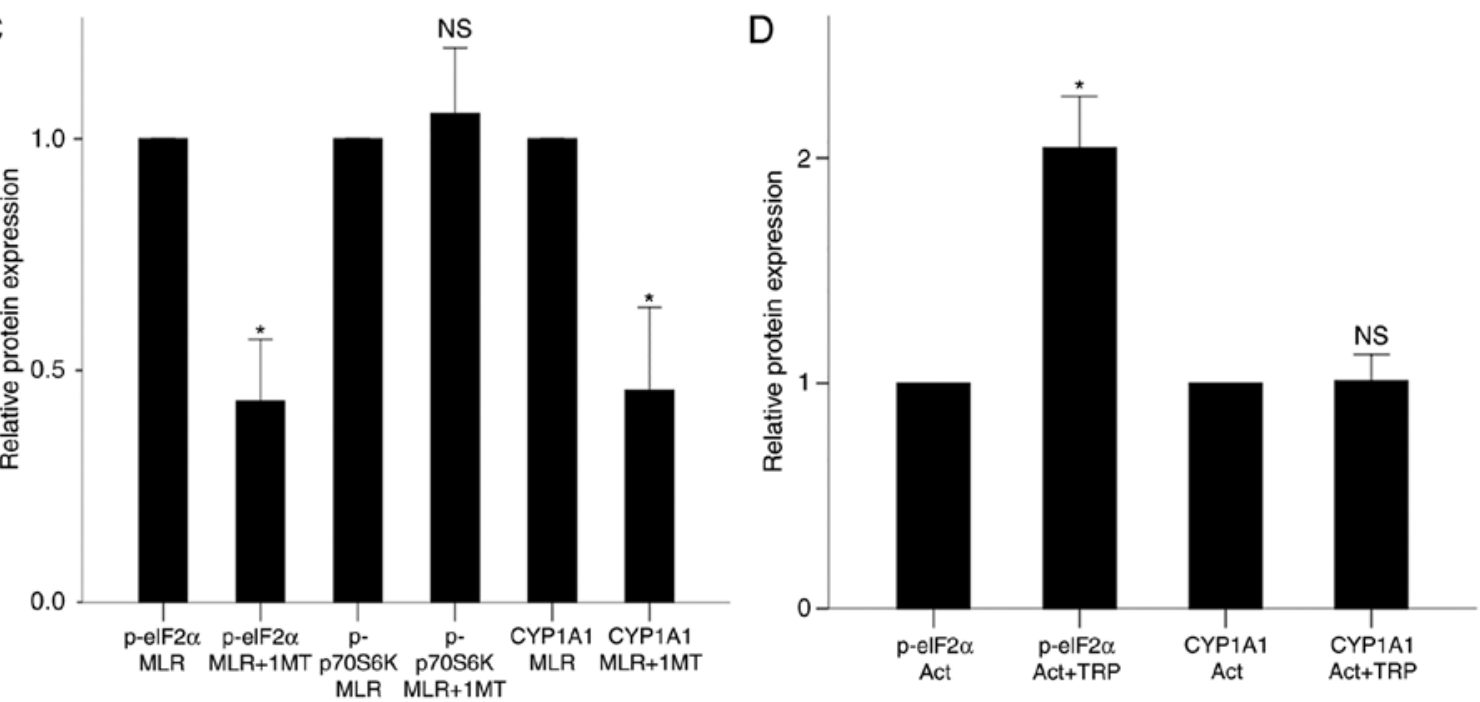

Figure 3. Effect of IDO on the activity of GCN2K, mTORC1 and AhR in MLR-derived CD4+ T-cells and effect of tryptophanol on the activity of GCN2K and AhR in activated isolated CD4+ T-cells. (A) PBMCs from different individuals were coupled in 10 different MLRs performed in the presence of oleate and treated with the IDO inhibitor 1-MT, then L-tryptophan consumption was measured. (B) CD4+ T-cells were isolated from the MLRs and protein was extracted for western blotting. Representative blots are shown for expression of p-eIF2 $\alpha$, p-p70S6K and CYP1A1 in the experimental groups. (C) Quantification of the western blot analysis results. 1-MT decreased the activities of GCN2K and AhR, assessed by the phosphorylation of the GCN2K substrate eIF2 $\alpha$ and the expression of the AhR transcriptional target CYP1A1, respectively. By contrast, 1-MT did not affect the activity of mTORC1, assessed by the phosphorylation of its substrate p70S6K. (D) CD4+ T-cells were isolated from the PBMCs of 6 individuals, cultured in an oleate-containing medium and activated with anti-CD2/CD3/CD28-conjugated beads in the presence or absence of the GCN2K activator tryptophanol. Quantification of the western blotting results from panel B. Tryptophanol increased GCN2K activity, assessed by the level of p-eIF2 $\alpha$, but it did not alter AhR activity, assessed by the expression of CYP1A1. Results are presented as mean \pm standard deviation. "P<0.05. IDO, indoleamine 2,3-dioxygenase; GCN2K, general control nonderepressible 2 kinase; mTORC1, mammalian target of rapamycin complex 1; AhR, aryl hydrocarbon receptor; MLR, mixed lymphocyte reactions; PBMCs, peripheral blood mononuclear cells; p-, phosphorylated; eIF2 $\alpha$, eukaryotic initiation factor $2 \alpha$; p70S6K, 70 kDa ribosomal protein S6 kinase; CYP1A1, cytochrome P450 family 1 subfamily A polypeptide 1; TRP, tryptophanol; NS, not significant.

In CD4+ T-cells derived from the above 1-MT-treated MLRs, the levels of the activated phosphorylated form of AMPK, as well as the levels of its substrate p-ACC2, decreased to $0.56 \pm 0.24(\mathrm{P}<0.001)$ and $0.33 \pm 0.14(\mathrm{P}<0.001)$ fold relative to the levels of the untreated cells, respectively (Fig. 6C and D). In isolated, cultured with oleate and activated with anti-CD2/CD3/CD28-conjugated beads CD4+ T-cells, tryptophanol increased p-AMPK and p-ACC2 levels by $1.45 \pm 0.11(\mathrm{P}<0.001)$ and $1.46 \pm 0.10(\mathrm{P}<0.001)$ fold relative to the untreated cells, respectively (Fig. $6 \mathrm{C}$ and E).

Finally, in CD4+ T-cells derived from MLRs performed in an oleate-containing medium and treated with 1-MT, the expression of all CPT1 isoenzymes was decreased compared with untreated cells (Fig. 6C). The protein expression levels of CPT1A decreased to $0.73 \pm 0.05(\mathrm{P}<0.001), \mathrm{CPT} 1 \mathrm{~B}$ to $0.54 \pm 0.13(\mathrm{P}<0.001)$ and $\mathrm{CPT} 1 \mathrm{C}$ to $0.42 \pm 0.12(\mathrm{P}<0.001)$ fold relative to the untreated cells (Fig. 6C and F). By contrast, in isolated, cultured with oleate and activated with anti-CD2/CD3/CD28-conjugated beads CD4+ T-cells, tryptophanol did not affect the expression of any of the CPT1 isoenzymes tested. CPT1A protein expression levels were at $1.05 \pm 0.22(\mathrm{P}=0.598), \mathrm{CPT} 1 \mathrm{~B}$ at $0.99 \pm 0.17(\mathrm{P}=0.933)$ and $\mathrm{CPT} 1 \mathrm{C}$ at $1.02 \pm 0.10(\mathrm{P}=0.706)$ fold relative to the untreated cells (Fig. 6C and G). In conclusion, these results 

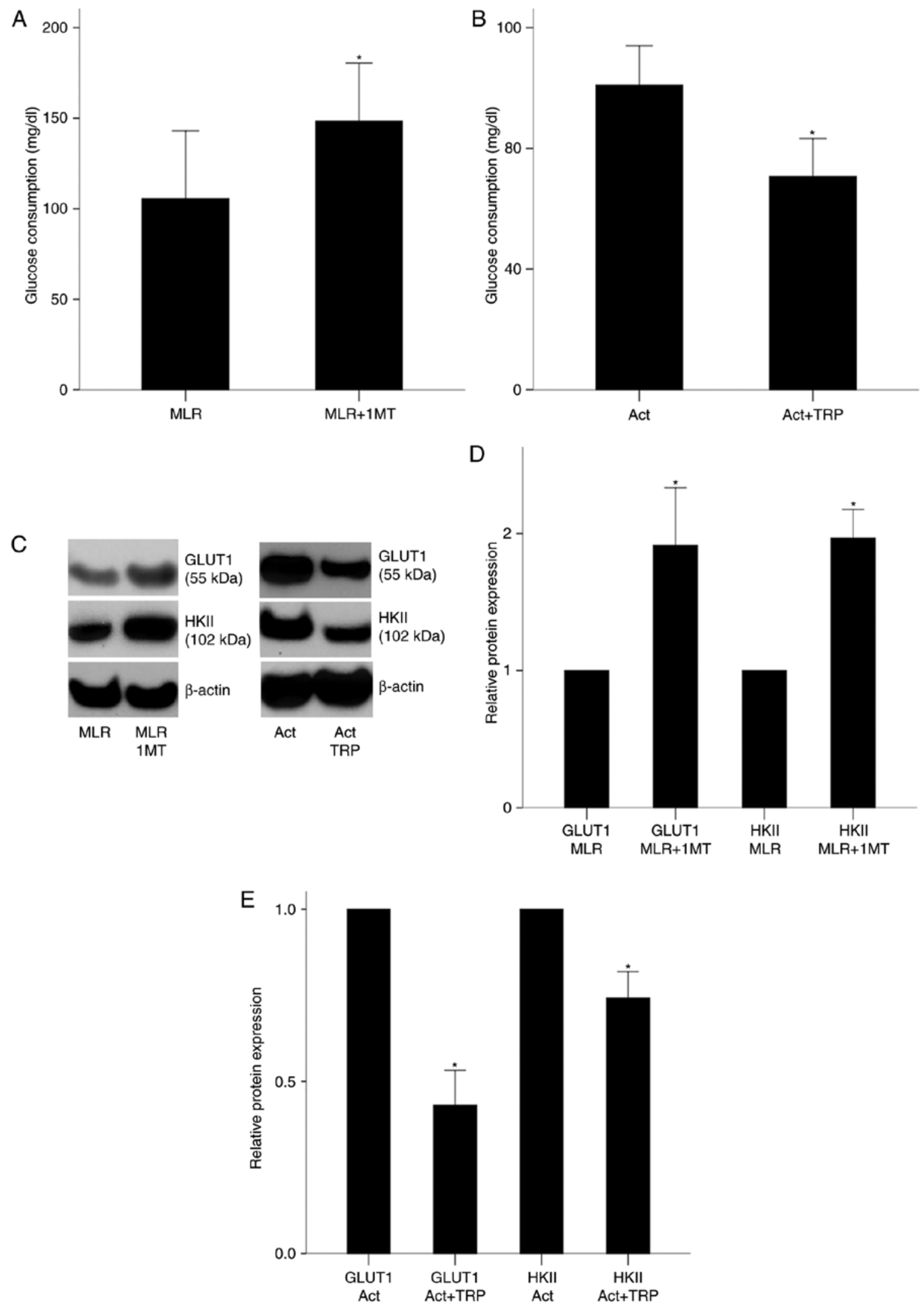

Figure 4. Effect of IDO on glucose consumption in MLRs and on GLUT1 and HKII expression in MLR-derived CD4+ T-cells, and effect of GCN2K activation on glucose consumption, GLUT1 and HKII expression in activated isolated CD4+ T-cells. (A) PBMCs from different individuals were coupled in 10 different MLRs performed in the presence of oleate, treated with the IDO inhibitor 1-MT, and their glucose consumption was measured. (B) CD4+ T-cells were isolated from the PBMCs of 6 individuals cultured in an oleate-containing medium, activated with anti-CD2/CD3/CD28-conjugated beads in the presence or absence of the GCN2K activator tryptophanol, and their glucose consumption was measured. (C) Representative blots of western blot analysis for GLUT1 and HKII protein expression levels in the experimental groups. (D) CD4+ T-cells were isolated from the MLRs and protein was extracted for western blotting. Quantification of results from panel C. 1-MT increased the expression of GLUT1 and HKII in MLR-derived CD4+ T-cells. (E) Quantification of results from panel C for the isolated and activated CD4+ T-cells. Tryptophanol decreased GLUT1 and HKII expression. Results are presented as mean \pm standard deviation. "P<0.05. IDO, indoleamine 2,3-dioxygenase; MLR, mixed lymphocyte reactions; GLUT1, glucose transporter-1; HKII, hexokinase-II; GCN2K, general control nonderepressible 2 kinase; PBMCs, peripheral blood mononuclear cells; TRP, tryptophanol; NS, not significant. 
A

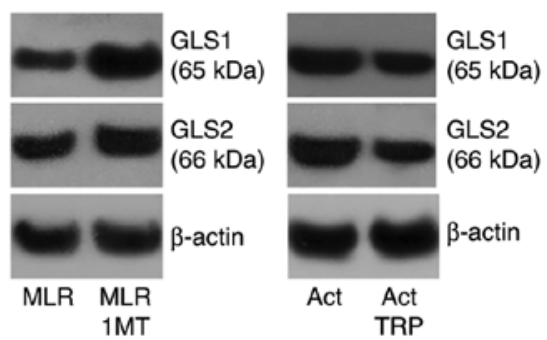

B

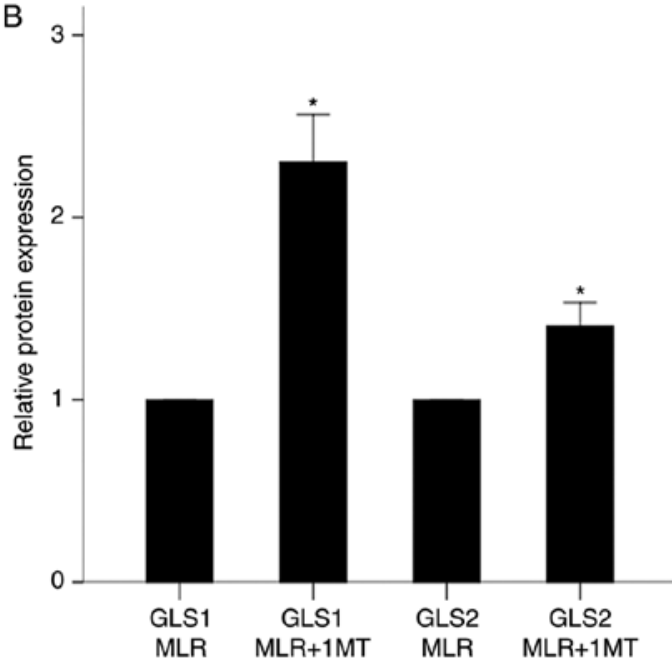

C

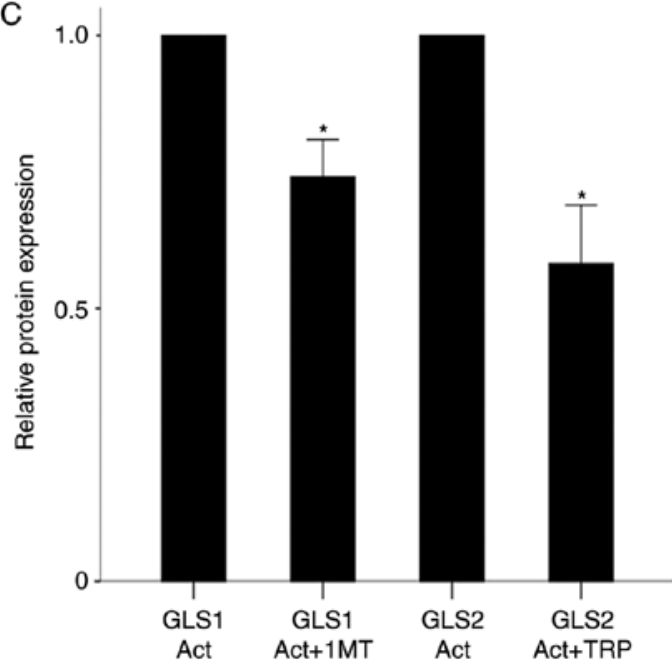

Figure 5. Effect of IDO inhibition and GCN2K activation on GLS1 and GLS2 expression in MLR-derived CD4+ T-cells and activated isolated CD4+ T-cells, respectively. (A) Representative blots from western blot analysis for GLS1 and GLS2 protein expression levels in the experimental groups. (B) Quantification of results from panel A. PBMCs from different individuals were coupled in 10 different MLRs performed in the presence of oleate and treated with the IDO inhibitor 1-MT. 1-MT increased the expression of both GLS1 and GLS2 in MLR-derived CD4+ T-cells. (C) Quantification of results from panel A. CD4+ T-cells were isolated from the PBMCs of 6 individuals, cultured in an oleate-containing medium and activated with anti-CD2/CD3/CD28-conjugated beads in the presence or absence of the GCN2K activator tryptophanol. Tryptophanol decreased GLS1 and GLS2 expression. Results are presented as mean \pm standard deviation. ${ }^{*} \mathrm{P}<0.05$. IDO, indoleamine 2,3-dioxygenase; GCN2K, general control nonderepressible 2 kinase; GLS, glutaminase; MLR, mixed lymphocyte reactions; PBMCs, peripheral blood mononuclear cells; TRP, tryptophanol; NS, not significant.

demonstrated that IDO activated AMPK and increased CPT1 expression, whereas the GCN2K activator tryptophanol, although it activated AMPK, did not significantly alter CPT1 expression.

\section{Discussion}

Because IDO has an important role in immune regulation, at least in part by altering T-cell metabolism $(3,4,7,8,13)$, the present study evaluated the effect of different IDO-induced pathways on glucose, glutamine, and free fatty acid metabolism and ultimately on CD4+ T-cell survival and proliferation.

Regarding cell proliferation in MLRs, the present study recapitulated the results of previous studies $(1-4,7,13)$. In MLRs performed in the commonly used culture medium, IDO decreased T-cell proliferation; however, when a free fatty acid was introduced in the culture medium, the IDO inhibitor 1-MT did not affect T-cell clonal expansion. By contrast, as is demonstrated in the present study for the first time, in activated isolated CD4+ T-cells, the presence of free fatty acids in the culture medium did not abrogate the anti-proliferative effect of the GCN2K activator tryptophanol. These results indicate that in the presence of free fatty acids the IDO-induced GCN2K activation is not responsible for the absence of the IDO antiproliferative effect on T-cells.

Similar results were obtained when apoptosis of CD4+ T-cells was assessed by examining the levels of activated cleaved caspase- 3 , in which all the apoptotic pathways converge (22). IDO increased apoptosis in CD4+ T-cells derived from MLRs performed in the commonly used culture medium, but when a free fatty acid was added into the culture medium the IDO inhibitor 1-MT did not affect cell apoptosis. These results are in agreement with the results of a previous study (7). By contrast, as the present study demonstrated for the first time, in activated isolated CD4+ T-cells, the presence of free fatty acids in the culture medium did not abolish the apoptotic effect of the GCN2K activator tryptophanol. These results indicate that other pathways, and not the GCN2K activation IDO-induced pathway, may be responsible for the absence of the IDO apoptotic effect on CD4+ T-cells when free fatty acids are present.

During an immune response, IDO affects T-cells by decreasing local L-tryptophan concentration, activating GCN2K and possibly inhibiting mTORC1 (1-5), as well as by producing kynurenine and activating AhR (6,7). In the present study, experiments were performed to assay the validity of our experimental systems regarding the above pathways and to define whether there is a role for mTORC1. In MLRs performed in the presence of oleate, IDO increased L-tryptophan consumption. In addition, in MLR-derived CD4+ T-cells, IDO activated GCN2K, assessed by the level of its phosphorylated substrate p-eIF2 $\alpha$ (23), but it did not affect mTORC1 activity, assessed by the level of its phosphorylated substrate p-p70S6K (24). This is in accordance with previous studies in which cell cultures were performed in the commonly used, fatty acid-free medium (1-4). Thus, the presence of free fatty acids does not alter the signal transduction pathways triggered by L-tryptophan depletion. No effect on mTORC1 activity was detected in the present study, in accordance with other studies reporting that mTORC1 is sensitive to the depletion of the amino acids leucine, isoleucine, valine and possibly arginine, but not of tryptophan (25). In addition to activation of 

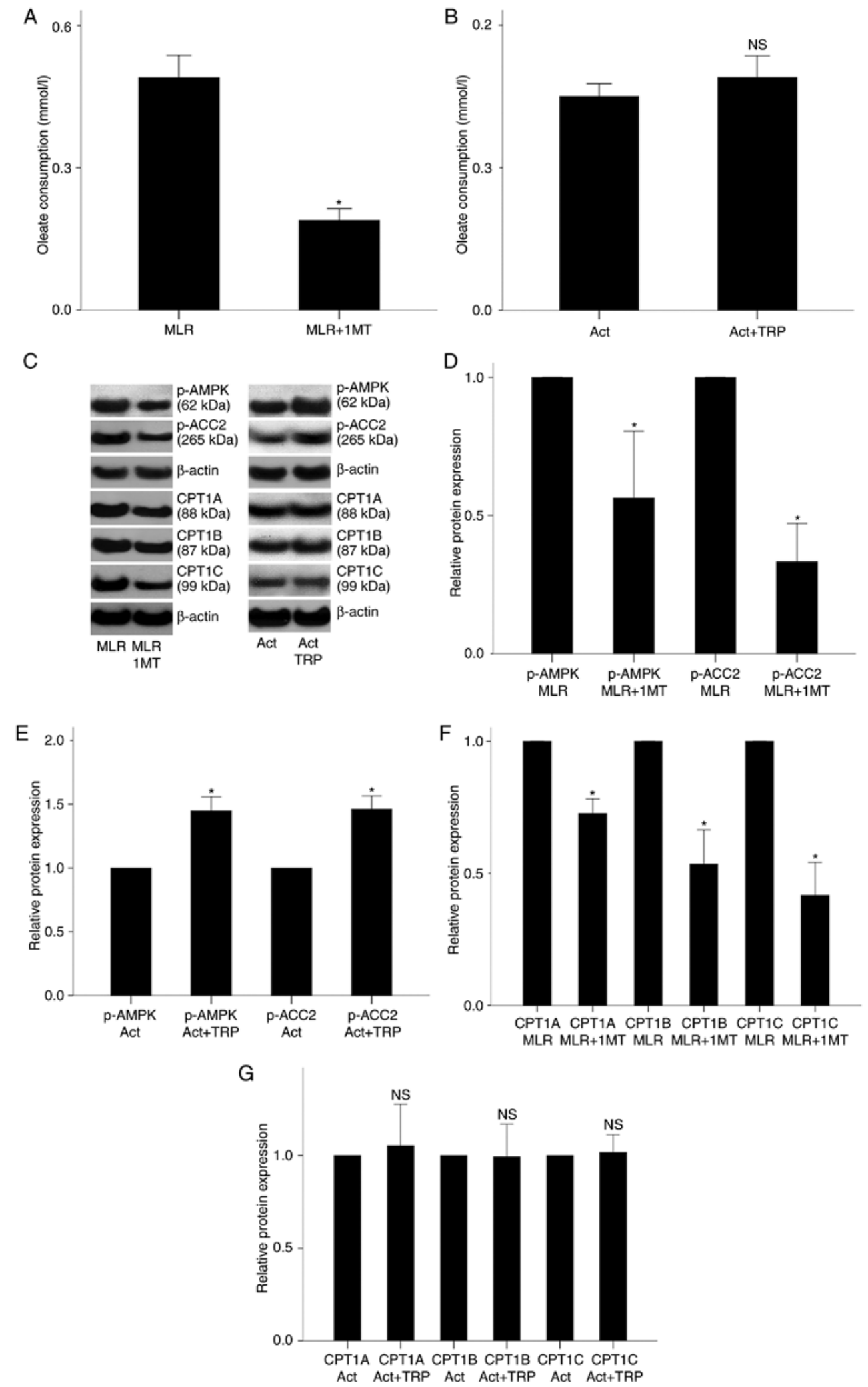

Figure 6. Effect of IDO on free fatty acid consumption in MLRs and on p-AMPK, p-ACC2 and CPT1 isoenzymes in MLR-derived CD4+ T-cells; and effect of GCN2K activation on free fatty acid consumption, and on p-AMPK, p-ACC2 and CPT1 isoenzymes in activated isolated CD4+ T-cells. (A) PBMCs from different individuals were coupled in 10 different MLRs performed in the presence of oleate, treated with the IDO inhibitor 1-MT, then their free fatty acid consumption was measured. (B) CD4+ T-cells were isolated from the PBMCs of 6 individuals cultured in an oleate-containing medium, activated with anti-CD2/CD3/CD28-conjugated beads in the presence or absence of the GCN2K activator tryptophanol, and then their free fatty acid consumption was measured. (C) Representative blots from western blot analysis in all experimental groups. (D) Quantification of results from panel C. In CD4+ T-cells isolated from the MLRs, 1-MT decreased the activity of AMPK, assessed by the level of its phosphorylated subunit $\alpha$ and the phosphorylation of its substrate ACC2. (E) Quantification of results from panel C. In isolated and activated CD4+ T-cells, tryptophanol increased p-AMPK and p-ACC2 levels. (F) Quantification of results from panel C. In CD4+ T-cells isolated from the MLRs, 1-MT decreased the expression of all CPT1 isoenzymes. (G) Quantification of results from panel C. In isolated and activated CD4+ T-cells, tryptophanol did not affect the expression of CPT1 isoenzymes. " $\mathrm{P}<0.05$. IDO, indoleamine 2,3-dioxygenase; MLR, mixed lymphocyte reactions; p-, phosphorylated; AMPK, AMP-activated protein kinase; ACC2, acetyl-CoA carboxylase 2; CPT1, carnitine palmitoyltransferase I; GCN2K, general control nonderepressible 2 kinase; PBMCs, peripheral blood mononuclear cells; TRP, tryptophanol; NS, not significant. 


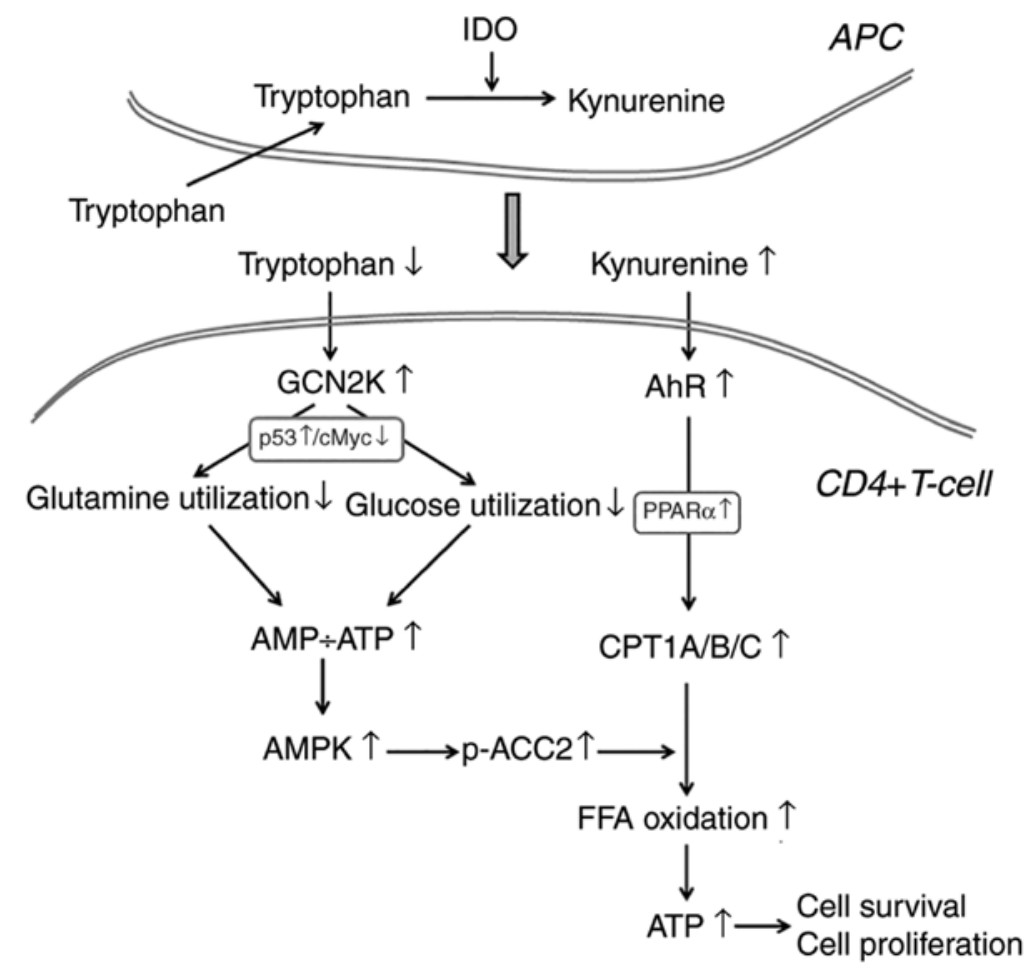

Figure 7. A proposed model regarding the effect of IDO on the utilization of the main energy sources in activated CD4+ T-cells. In the immune response microenvironment, IDO, by degrading L-tryptophan along the kynurenine pathway, activates GCN2K and AhR. Activation of GCN2K, possibly by upregulating p53 and downregulating c-Myc, leads to decreased utilization of glucose and glutamine, two pivotal sources of energy in activated CD4+ T-cells, resulting in reduced ATP production. The latter activates AMPK, which phosphorylates and inactivates ACC2, resulting in decreased production of the CPT1 inhibitor malonyl-CoA. In parallel, activation of AhR, possibly by upregulating PPAR $\alpha$, increases the expression of all CPT1 isoenzymes. Since CPT1 controls free fatty acid oxidation, these IDO-induced alterations promote free fatty acid oxidation as an alternative fuel for ATP production, supplying the required energy for CD4+ T-cell survival and proliferation. The steps in the present model that are based on findings from previously published studies are noted in gray font. IDO, indoleamine 2,3-dioxygenase; GCN2K, general control nonderepressible 2 kinase; AhR, aryl hydrocarbon receptor; AMPK, AMP-activated protein kinase; ACC2, acetyl-CoA carboxylase 2; CPT1, carnitine palmitoyltransferase I; PPAR $\alpha$, peroxisome proliferator-activated receptor- $\alpha$.

GCN2K, in MLR-derived CD4+ T-cells, IDO activated AhR, assessed by the level of its transcriptional target CYP1A1 (26). As expected, in isolated CD4+ T-cells cultured and activated in an oleate-containing medium, tryptophanol activated GCN2K, but not AhR.

Consistent with results obtained from MLRs in a fatty acid-free medium $(3,13)$, the present study demonstrated that glycolysis was also decreased by IDO in the presence of oleate. In addition, the expression of GLUT1, the main glucose transporter in CD4+ T-cells (11), as well as of the first enzyme of the glycolytic pathway HKII, decreased by IDO in MLR-derived CD4+ T-cells. These results were recapitulated by tryptophanol in isolated CD4+ T-cells cultured with oleate and activated with anti-CD2/CD3/CD28 beads. Thus, it is likely that IDO through activation of GCN2K starves CD4+ T-cells from glucose.

In addition to glucose, another energy source for activated CD4+ T-cells is glutamine $(9,10)$. However, as in MLRs performed in a fatty acid-free medium $(3,4,13)$, the present study demonstrated that, in the presence of oleate, IDO decreased the expression of both GLS isoenzymes in MLR-derived CD4+ T-cells. This was also confirmed following tryptophanol treatment in isolated CD4+ T-cells cultured and activated in an oleate-containing medium. Hence, it can be hypothesized that IDO, through activation of GCN2K, starves CD4+ T-cells of glutamine, as well as glucose.
Regarding the third available energy source, the free fatty acids, in MLRs, IDO increased oleate consumption, which is in agreement with a previous study (7). By contrast, in isolated and activated CD4+ T-cells, tryptophanol did not affect oleate consumption, which remained at very low levels, precluding a role for GCN2K in the IDO-induced increase in fatty acid consumption. Therefore, the ability of IDO to increase free fatty acid consumption in CD4+ T-cells could be attributed to the activation of AhR. This alternative source of energy appears to compensate for the decreased glucose and glutamine utilization and to provide the required energy for cell survival and proliferation.

Next, the molecular mechanisms that may be involved in the IDO-induced increase in free fatty acid oxidation were investigated in CD4+ T-cells. The IDO-induced and GCN2K-mediated decrease in glucose and glutamine utilization would be expected to decrease ATP production in CD4+ T-cells resulting in activation of AMPK. Indeed, the present results demonstrated that IDO increased the activated form of AMPK in MLR-derived CD4+ T-cells, and tryptophanol had similar effect in isolated and activated CD4+ T-cells. Once activated, AMPK phosphorylates and inactivates ACC2, which catalyzes the carboxylation of acetyl-CoA to the CPT1 inhibitor malonyl-CoA (27-29). Accordingly, the present results demonstrated that IDO increased p-ACC2 levels in MLR-derived CD4+ T-cells, and tryptophanol had the same effect in isolated and activated CD4+ T-cells. Since 
CPT1 controls free fatty acid entry into the mitochondria for oxidation $(28,29)$, it would be expected that inactivation of ACC2 by AMPK would release CPT1 activity and increase free fatty acid oxidation. However, although IDO activated AMPK and inactivated ACC2 in MLR-derived CD4+ T-cells, similar to tryptophanol in isolated, activated CD4+ T-cells, free fatty acid consumption increased only in MLRs. Thus, an additional molecular mechanism, specifically mediated by AhR may be responsible for the IDO-induced increase in fatty acid oxidation.

Activation of AhR increases the expression of the transcription factor peroxisome proliferator-activated receptor- $\alpha$ (PPAR- $\alpha$ ) (30), which is known to upregulate the expression of all CPT1 isoenzymes (31-33). Therefore, the expression levels of the isoenzymes CPT1A, CPT1B, and CPT1C were evaluated in the present experimental systems. In MLR-derived CD4+ T-cells, IDO increased the expression of all CPT1 isoenzymes, whereas, in activated isolated CD4+ T-cells, tryptophanol did not alter their expression. These results indicate that IDO may have increased CPT1 expression through activation of AhR. It is likely that through this molecular mechanism IDO increases free fatty acid consumption in CD4+ T-cells.

The present results support that free fatty acid oxidation compensates for decreased glucose and glutamine utilization in CD4+ T-cells providing the required energy for cell survival and proliferation. On the other hand, IDO-induced and AhR-mediated increase in free fatty acid oxidation may enhance CD4+ T-cell differentiation to Tregs. Inhibition of fatty acid oxidation with the CPT1 inhibitor etomoxir abrogates differentiation of naïve CD4+ T-cells to Tregs (12). Notably, activation of AhR promotes Treg differentiation (6), activation of PPAR- $\alpha$ ameliorates the course of autoimmune diseases (34-37), and PPAR- $\alpha$ activity is required for Treg differentiation and suppressive function (38-40). Finally, it is possible that a portion of free fatty acids is consumed by the expanded CD4+ T-cells as building blocks, since IDO by activating GCN2K decreases fatty acid synthesis (4), which is required for T-cell proliferation (41). This, along with the decreased apoptosis, may contribute to the increased cell proliferation index when MLRs were performed in a free fatty acid-containing culture medium as observed in the present study.

Collectively, the present results propose the following model regarding the effect of IDO on the utilization of the main energy sources in activated CD4+ T-cells. As depicted in Fig. 7, in the immune response microenvironment, IDO, by degrading L-tryptophan along the kynurenine pathway, activates GCN2K and AhR. Activation of GCN2K, possibly by upregulating p53 and downregulating c-Myc, leads to decreased glycolysis and glutaminolysis, two main energy sources in activated CD4+ T-cells, resulting in reduced ATP production. The latter activates AMPK, which phosphorylates and inactivates ACC2 resulting in decreased production of the CPT1 inhibitor malonyl-CoA. In parallel, activation of AhR, possibly by upregulating PPAR $\alpha$, increases the expression of all CPT1 isoenzymes. Since CPT1 controls free fatty acid oxidation, these IDO-induced alterations promote free fatty acid oxidation as an alternative fuel for ATP production, supplying the required energy for CD4+ T-cell survival and proliferation.
In conclusion, IDO decreases glycolysis and glutaminolysis by activating GCN2K, while it increases free fatty acid oxidation by activating AhR, providing the necessary energy for CD4+ T-cell survival and proliferation. Thus, contrary to what is generally hypothesized to date, in a normal environment that contains fatty acids, the immunosuppressive effect of IDO cannot be attributed to a decrease in CD4+ T-cell proliferation and survival.

\section{Acknowledgements}

Not applicable.

\section{Funding}

No funding was received.

\section{Availability of data and materials}

The analyzed datasets generated during the study are available from the corresponding author on reasonable request.

\section{Author's contributions}

TE, GP and IS designed the study, TE and GP performed the experiments, TE, GP, VL and IS interpreted the results, TE and GP wrote the manuscript. All authors read and approved the final manuscript.

\section{Ethics approval and consent to participate}

A written informed consent was obtained from each individual enrolled in the study. The Ethics Committee of the Faculty of Medicine, University of Thessaly (Larisa, Greece) approved the study protocol (approval no. 558/10-2-2017).

\section{Consent for publication}

Not applicable.

\section{Competing interests}

The authors declare that they have no competing interests.

\section{References}

1. Munn DH, Sharma MD, Baban B, Harding HP, Zhang Y, Ron D and Mellor AL: GCN2 kinase in T cells mediates proliferative arrest and anergy induction in response to indoleamine 2,3-dioxygenase. Immunity 22: 633-642, 2005.

2. Eleftheriadis T, Pissas G, Yiannaki E, Markala D, Arampatzis S, Antoniadi G, Liakopoulos V and Stefanidis I: Inhibition of indoleamine 2,3-dioxygenase in mixed lymphocyte reaction affects glucose influx and enzymes involved in aerobic glycolysis and glutaminolysis in alloreactive T-cells. Hum Immunol 74: 1501-1509, 2013

3. Eleftheriadis T, Pissas G, Antoniadi G, Spanoulis A, Liakopoulos V and Stefanidis I: Indoleamine 2,3-dioxygenase increases p53 levels in alloreactive human T cells, and both indoleamine 2,3-dioxygenase and p53 suppress glucose uptake, glycolysis and proliferation. Int Immunol 26: 673-684, 2014.

4. Eleftheriadis T, Pissas G, Antoniadi G, Liakopoulos V and Stefanidis I: Indoleamine 2,3-dioxygenase depletes tryptophan, activates general control non-derepressible 2 kinase and down-regulates key enzymes involved in fatty acid synthesis in primary human $C D 4^{+} T$ cells. Immunology 146: 292-300, 2015. 
5. Cobbold SP, Adams E, Farquhar CA, Nolan KF, Howie D, Lui KO, Fairchild PJ, Mellor AL, Ron D and Waldmann H: Infectious tolerance via the consumption of essential amino acids and mTOR signaling. Proc Natl Acad Sci USA 106: 12055-12060, 2009.

6. Mezrich JD, Fechner JH, Zhang X, Johnson BP, Burlingham WJ and Bradfield CA: An interaction between kynurenine and the aryl hydrocarbon receptor can generate regulatory $\mathrm{T}$ cells. J Immunol 185: 3190-3198, 2010.

7. Eleftheriadis T, Pissas G, Sounidaki M, Tsogka K, Antoniadis N, Antoniadi G, Liakopoulos V and Stefanidis I: Indoleamine 2,3-dioxygenase, by degrading L-tryptophan, enhances carnitine palmitoyltransferase I activity and fatty acid oxidation, and exerts fatty acid-dependent effects in human alloreactive CD4 ${ }^{\times}$-cells. Int J Mol Med 38: 1605-1613, 2016.

8. Curti A, Trabanelli S, Salvestrini V, Baccarani M and Lemoli RM: The role of indoleamine 2,3-dioxygenase in the induction of immune tolerance: Focus on hematology. Blood 113 : 2394-2401, 2009.

9. Wang R, Dillon CP, Shi LZ, Milasta S, Carter R, Finkelstein D, McCormick LL, Fitzgerald P, Chi H, Munger J and Green DR: The transcription factor Myc controls metabolic reprogramming upon T lymphocyte activation. Immunity 35: 871-882, 2011.

10. Caro-Maldonado A, Gerriets VA and Rathmell JC: Matched and mismatched metabolic fuels in lymphocyte function. Semin Immunol 24: 405-413, 2012.

11. Macintyre AN, Gerriets VA, Nichols AG, Michalek RD Rudolph MC, Deoliveira D, Anderson SM, Abel ED, Chen BJ, Hale LP and Rathmell JC: The glucose transporter Glut1 is selectively essential for CD4 T cell activation and effector function. Cell Metab 20: 61-72, 2014

12. Michalek RD, Gerriets VA, Jacobs SR, Macintyre AN, MacIver NJ, Mason EF, Sullivan SA, Nichols AG and Rathmell JC: Cutting edge: Distinct glycolytic and lipid oxidative metabolic programs are essential for effector and regulatory $\mathrm{CD}^{+} \mathrm{T}$ cell subsets. J Immunol 186: 3299-3303, 2011.

13. Eleftheriadis T, Pissas G, Antoniadi G, Tsogca K, Sounidaki M, Liakopoulos V and Stefanidis I: Indoleamine 2,3-dioxygenase downregulates $\mathrm{T}$-cell receptor complex $\zeta$-chain and c-Myc, and reduces proliferation, lactate dehydrogenase levels and mitochondrial glutaminase in human T-cells. Mol Med Rep 13 925-932, 2015.

14. Gurtner GJ, Newberry RD, Schloemann SR, McDonald KG and Stenson WF: Inhibition of indoleamine 2,3-dioxygenase augments trinitrobenzene sulfonic acid colitis in mice. Gastroenterology 125: 1762-1773, 2003.

15. Kwidzinski E, Bunse J, Aktas O, Richter D, Mutlu L, Zipp F, Nitsch R and Bechmann I: Indolamine 2,3-dioxygenase is expressed in the CNS and down-regulates autoimmune inflammation. FASEB J 19: 1347-1349, 2005.

16. Alexander AM, Crawford M, Bertera S, Rudert WA, Takikawa O, Robbins PD and Trucco M: Indoleamine 2,3-dioxygenase expression in transplanted NOD Islets prolongs graft survival after adoptive transfer of diabetogenic splenocytes. Diabetes 51: 356-365, 2002

17. Li Y, Tredget EE, Ghaffari A, Lin X, Kilani RT and Ghahary A Local expression of indoleamine 2,3-dioxygenase protects engraftment of xenogeneic skin substitute. J Invest Dermatol 126: 128-136, 2006

18. Munn DH, Zhou M, Attwood JT, Bondarev I, Conway SJ, Marshall B, Brown C and Mellor AL: Prevention of allogeneic fetal rejection by tryptophan catabolism. Science 281: 1191-1193, 1998.

19. Munn DH and Mellor AL: Indoleamine 2,3-dioxygenase and tumor-induced tolerance. J Clin Invest 117: 1147-1154, 2007.

20. Sato T, Deiwick A, Raddatz G, Koyama K and Schlitt HJ: Interactions of allogeneic human mononuclear cells in the two-way mixed leucocyte culture (MLC): Influence of cell numbers, subpopulations and cyclosporin. Clin Exp Immunol 115: 301-308, 1999.

21. Staiger K, Staiger H, Weigert C, Haas C, Häring HU and Kellerer M: Saturated, but not unsaturated, fatty acids induce apoptosis of human coronary artery endothelial cells via nuclear factor-kappaB activation. Diabetes 55: 3121-3126, 2006.

22. Fadeel B and Orrenius S: Apoptosis: A basic biological phenomenon with wide-ranging implications in human disease. J Intern Med 258: 479-517, 2005.
23. Castilho BA, Shanmugam R, Silva RC, Ramesh R, Himme BM and Sattlegger E: Keeping the eIF2 alpha kinase Gcn2 in check. Biochim Biophys Acta 1843: 1948-1968, 2014.

24. Ma XM and Blenis J: Molecular mechanisms of mTOR-mediated translational control. Nat Rev Mol Cell Biol 10: 307-318, 2009.

25. Gallinetti J, Harputlugil E and Mitchell JR: Amino acid sensing in dietary-restriction-mediated longevity: Roles of signal-transducing kinases GCN2 and TOR. Biochem J 449: $1-10,2013$.

26. Ma Q: Induction of CYP1A1. The AhR/DRE paradigm: Transcription, receptor regulation, and expanding biological roles. Curr Drug Metab 2: 149-164, 2001.

27. Mihaylova MM and Shaw RJ: The AMPK signalling pathway coordinates cell growth, autophagy and metabolism. Nat Cell Biol 13: 1016-1023, 2011.

28. Lopaschuk GD, Ussher JR, Folmes CD, Jaswal JS and Stanley WC: Myocardial fatty acid metabolism in health and disease. Physiol Rev 90: 207-258, 2010.

29. Schreurs M, Kuipers F and van der Leij FR: Regulatory enzymes of mitochondrial beta-oxidation as targets for treatment of the metabolic syndrome. Obes Rev 11: 380-388, 2010.

30. Wang C, Xu CX, Krager SL, Bottum KM, Liao DF and Tischkau SA: Aryl hydrocarbon receptor deficiency enhances insulin sensitivity and reduces PPAR- $\alpha$ pathway activity in mice. Environ Health Perspect 119: 1739-1744, 2011.

31. Song S, Attia RR, Connaughton S, Niesen MI, Ness GC, Elam MB, Hori RT, Cook GA and Park EA: Peroxisome proliferator activated receptor alpha (PPARalpha) and PPAR gamma coactivator (PGC-1alpha) induce carnitine palmitoyltransferase IA (CPT-1A) via independent gene elements. Mol Cell Endocrinol 325: 54-63, 2010

32. Kok BP, Dyck JR, Harris TE and Brindley DN: Differential regulation of the expressions of the PGC-1 $\alpha$ splice variants, lipins, and PPAR $\alpha$ in heart compared to liver. J Lipid Res 54: 1662-1677, 2013.

33. Chen Y, Wang Y, Huang Y, Zeng H, Hu B, Guan L, Zhang H, $\mathrm{Yu}$ AM, Johnson $\mathrm{CH}$, Gonzalez FJ, et al: PPAR $\alpha$ regulates tumor cell proliferation and senescence via a novel target gene carnitine palmitoyltransferase 1C. Carcinogenesis 38: 474-483, 2017.

34. Dunn SE, Ousman SS, Sobel RA, Zuniga L, Baranzini SE, Youssef S, Crowell A, Loh J, Oksenberg J and Steinman L: Peroxisome proliferator-activated receptor (PPAR)alpha expression in $\mathrm{T}$ cells mediates gender differences in development of T cell-mediated autoimmunity. J Exp Med 204: 321-330, 2007.

35. Lee JW, Bajwa PJ, Carson MJ, Jeske DR, Cong Y, Elson CO, Lytle C and Straus DS: Fenofibrate represses interleukin-17 and interferon-gamma expression and improves colitis in interleukin-10-deficient mice. Gastroenterology 133: 108-123, 2007.

36. Gocke AR, Hussain RZ, Yang Y, Peng H, Weiner J, Ben LH, Drew PD, Stuve O, Lovett-Racke AE and Racke MK: Transcriptional modulation of the immune response by peroxisome proliferator-activated receptor-\{alpha\} agonists in autoimmune disease. J Immunol 182: 4479-4487, 2009.

37. Azuma YT, Nishiyama K, Matsuo Y, Kuwamura M, Morioka A, Nakajima $\mathrm{H}$ and Takeuchi T: PPAR $\alpha$ contributes to colonic protection in mice with DSS-induced colitis. Int Immunopharmacol 10: $1261-1267,2010$

38. Kim MS, Pyun HB and Hwang JK: Panduratin A, an activator of PPAR- $\alpha / \delta$, suppresses the development of oxazolone-induced atopic dermatitis-like symptoms in hairless mice. Life Sci 100: 45-54, 2014.

39. Lei J, Hasegawa H, Matsumoto T and Yasukawa M: Peroxisome proliferator-activated receptor $\alpha$ and $\gamma$ agonists together with TGF- $\beta$ convert human $\mathrm{CD} 4^{+} \mathrm{CD} 25^{-} \mathrm{T}$ cells into functional Foxp3+ regulatory T cells. J Immunol 185: 7186-7198, 2010.

40. Hichami A, Yessoufou A, Ghiringhelli F, Salvadori F, Moutairou K, Zwetyenga N and Khan NA: Peroxisome proliferator-activated receptor alpha deficiency impairs regulatory $\mathrm{T}$ cell functions: Possible application in the inhibition of melanoma tumor growth in mice. Biochimie 131: 1-10, 2016.

41. Berod L, Friedrich C, Nandan A, Freitag J, Hagemann S, Harmrolfs K, Sandouk A, Hesse C, Castro CN, Bähre H, et al: De novo fatty acid synthesis controls the fate between regulatory $\mathrm{T}$ and T helper 17 cells. Nat Med 20: 1327-1333, 2014. 